\title{
Head and neck CT angiography to assess the internal carotid artery stealing pathway
}

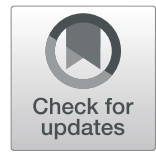

\author{
Dongxu Wang ${ }^{1} \mathbb{B}$, Zheng Li', Xiaoyang Zheng ${ }^{1}$, Houyi Cong' ${ }^{1}$, Tianyu Zhang ${ }^{1}$, Zhenghua Wang ${ }^{2}$, \\ Yuguang Wang ${ }^{1 *}$ and Jun $\mathrm{He}^{3}$
}

\begin{abstract}
Background: Common carotid artery occlusive disease (CCAOD) could form internal carotid artery steal pathways. Based on the diagnostic results of digital subtraction angiography (DSA), head and neck computed tomography angiography (CTA) was used to find the internal carotid artery stealing pathway after CCAOD. Methods: The clinical and imaging data of 18 patients with CCAOD were retrospectively analyzed. DSA and CTA was used to evaluate internal carotid artery steal pathways.
\end{abstract}

Results: Of the 18 patients with CCAOD, 10 patients found internal carotid artery steal pathways. There were 7 males and 3 females. Vascular ultrasound examination of all patients: The affected side had no blood flow in common carotid artery (CCA), and had retrograde blood flow in the external carotid artery (ECA). The blood flow of the affected side was decreased in the internal carotid artery (ICA), but it was antegrade. DSA diagnosed 10 cases of CCA occlusion and CTA diagnosed 10 cases of CCA occlusion. DSA and CTA found 6 internal carotid artery blood stealing pathways: (1) Vertebral artery $\rightarrow$ occipital artery $\rightarrow$ external carotid artery $\rightarrow$ internal carotid artery (6 cases); (2) Thyrocervical trunk $\rightarrow$ ascending cervical artery $\rightarrow$ occipital artery $\rightarrow$ external carotid artery $\rightarrow$ internal carotid artery (7 cases); (3) Costocervical trunk $\rightarrow$ deep cervical artery $\rightarrow$ occipital artery $\rightarrow$ external carotid artery $\rightarrow$ internal carotid artery (6 cases); (4) Affected side thyroid neck trunk $\rightarrow$ inferior thyroid artery $\rightarrow$ superior thyroid artery $\rightarrow$ external carotid artery $\rightarrow$ internal carotid artery (2 cases); (5) Contralateral external carotid artery $\rightarrow$ contralateral superior thyroid artery $\rightarrow$ affected superior thyroid artery $\rightarrow$ external carotid artery $\rightarrow$ neck Internal artery (2 cases); (6) Parathyroid neck $\rightarrow$ superficial cervical artery $\rightarrow$ occipital artery $\rightarrow$ external carotid artery $\rightarrow$ internal carotid artery (1 case).

Conclusions: The patients with CCAOD can find the internal carotid artery blood stealing pathway through CTA.

Keywords: Computed tomography angiography, Stroke, Common carotid artery, Artery occlusive, Internal carotid artery, Steal blood

\section{Background}

Common carotid artery occlusive disease (CCAOD) refers to severe stenosis (stenosis $\geq 80 \%$ ), sub occlusion (stenosis $\geq 95 \%$ ) and occlusion caused by various causes, which can cause ischemic stroke [1]. Cerebral blood flow compensation is more complicated with CCAOD. When

\footnotetext{
*Correspondence: wangdongxu19840312@163.com

'Departments of CT, The Second Affiliated Hospital of Qiqihar Medical College, 37 West Zhonghua Road, Qiqihar, Heilongjiang 161006, PR China Full list of author information is available at the end of the article
}

CCAOD occurs and the internal and external carotid arteries are unobstructed, the internal carotid artery pressure at the distal end of the lesion is significantly lower than that of the ipsilateral external carotid artery. The direction of blood flow in the artery is reversed, which is called "internal carotid artery steal" [2, 3]. The establishment of collateral circulation can relieve the symptoms of cerebral ischemia. The assessment of collateral circulation pathways in ischemic stroke is currently being actively studied. Due to the low

(c) The Author(s). 2020 Open Access This article is licensed under a Creative Commons Attribution 4.0 International License, which permits use, sharing, adaptation, distribution and reproduction in any medium or format, as long as you give appropriate credit to the original author(s) and the source, provide a link to the Creative Commons licence, and indicate if changes were made. The images or other third party material in this article are included in the article's Creative Commons licence, unless indicated otherwise in a credit line to the material. If material is not included in the article's Creative Commons licence and your intended use is not permitted by statutory regulation or exceeds the permitted use, you will need to obtain permission directly from the copyright holder. To view a copy of this licence, visit http://creativecommons.org/licenses/by/4.0/ The Creative Commons Public Domain Dedication waiver (http://creativecommons.org/publicdomain/zero/1.0/) applies to the data made available in this article, unless otherwise stated in a credit line to the data. 
incidence of CCAOD, few reports of blood stealing pathways have been reported.

There are many imaging methods for assessing internal carotid blood stealing in CCAOD [4]. Digital subtraction angiography (DSA) is the gold standard, but DSA is an invasive inspection method and expensive, and cannot be widely used for clinical screening [5]. Computed tomography angiography (CTA) is a non-invasive examination with high accuracy, and its consistency with DSA is $97 \%$ $[6,7]$. Based on the diagnostic results of DSA, CTA was used to find the internal carotid artery theft pathway in CCAOD patients. Understanding blood stealing pathways is a prerequisite for personalized treatment of stroke patients.

\section{Methods}

The study protocol was approved by the Regional Ethics Committee for Clinical Research of the Second Affiliated Hospital of Qiqihar Medical College. The study obtained informed consent from all patients. We retrospectively analyzed 4585 patients with ischemic stroke diagnosed in the Cerebral Vascular Stenosis Diagnosis and Intervention Center of the Second Affiliated Hospital of Qiqihar Medical College from December 2015 to December 2019. 18 cases were CCAOD, of which 10 cases had internal carotid blood stealing. There were 7 males and 3 females, aged 51 to 77 years, with an average of 63.6 years.

\section{Head and neck CTA imaging protocol}

All patients were scanned with a 64-slice CT (Toshiba, Aquillion). The patient was supine with her/his head stopped and swallowing was prohibited during the scan. The scanning range was from the ascending aorta to the top of the skull. Using plain scan and enhanced scan schemes. The scanning parameters are as follows: The tube voltage was $120 \mathrm{kV}$, the tube current was $200 \mathrm{~mA}$, the pitch was 41.0, and the scanning layer thickness was $0.5 \mathrm{~mm}$; The enhanced scan parameters are as follows: The tube voltage was $120 \mathrm{kV}$, the tube current was 300 $\mathrm{mA}$, the pitch was 41.0 , and the scanning layer thickness was $0.5 \mathrm{~mm}$. A double-barreled high-pressure syringe (USA, Mallinckrodt) was used to inject the contrast (Ultravist, $100 \mathrm{~mL}$ ) $60 \sim 80 \mathrm{ml}$ through the median vein of right elbow, and the injection rate was $5.0 \mathrm{~mL} / \mathrm{s}$. Immediately after the injection of contrast agent, $40 \mathrm{~mL}$ of saline was injected at a rate of $5.0 \mathrm{~mL} / \mathrm{s}$. The 5-6th cervical spine plane was used as the monitoring plane, and the left common carotid artery was monitored by ROI. The threshold was set to $120 \mathrm{HU}$, and scanning was triggered automatically after $8 \mathrm{~s}$.

The CT image data was transmitted to a postprocessing workstation. The two scanned images were deboned, subtracted, trimmed, and rotated. The image display method is demonstrated using volume rendering
(VR), multi-planner reformation (MPR), maximum intensity projection (MIP), and curved planar reconstruction (CPR) .

Evaluation and Data Analysis.

All head and neck CTA images were evaluated by two experienced physicians, and the degree of vascular stenosis and collateral circulation pathways were judged. If the opinions were not consistent, a consensus decision was made after discussion.

\section{Neck vascular ultrasound examination}

Neck vascular ultrasound was performed in all patients. A $4 \mathrm{MHz}$ pulsed probe was used to detect the blood flow of both common carotid artery (CCA), internal carotid artery (ICA) and external carotid artery (ECA).

\section{DSA examination}

All patients were examined by digital subtraction angiography (GE, Healthcare LCE) and high pressure syringe (Toshiba, Nemoto). In supine position, the patients were disinfected and covered with cloth in the conventional operation area. After local anesthesia, Seldinger puncture of femoral artery was performed, and angiography of aortic arch and vessels above aortic arch was performed respectively. The obtained image is processed by digitization as the gold standard of diagnosis.

\section{Results}

18 CCAOD patients accounted for $0.39 \%$ of patients with ischemic stroke, of which 10 patients had internal carotid blood stealing (Table 1). Risk factors: There were 8 cases of hypertension, 7 cases of dyslipidemia, 3 cases of diabetes mellitus, 5 cases of smoking and 2 cases of drinking. Neck vascular ultrasound examination of all patients: The affected side had no blood flow in CCA, and had retrograde blood flow in the ECA. The blood flow of the affected side was decreased in the ICA, but it was antegrade. DSA diagnosed 10 cases of CCA occlusion and CTA diagnosed 10 cases of CCA occlusion. CTA and DSA found 6 internal carotid artery blood stealing pathways (Figs. 1, 2, and 3): (1) Vertebral artery $\rightarrow$ occipital artery $\rightarrow$ external carotid artery $\rightarrow$ internal carotid artery (6 cases); (2) Thyrocervical trunk $\rightarrow$ ascending cervical artery $\rightarrow$ occipital artery $\rightarrow$ external carotid artery $\rightarrow$ internal carotid artery (7 cases); (3) Costocervical trunk $\rightarrow$ deep cervical artery $\rightarrow$ occipital artery $\rightarrow$ external carotid artery $\rightarrow$ internal carotid artery (6 cases); (4) Affected side thyroid neck trunk $\rightarrow$ inferior thyroid artery $\rightarrow$ superior thyroid artery $\rightarrow$ external carotid artery $\rightarrow$ internal carotid artery (2 cases); (5) Contralateral external carotid artery $\rightarrow$ contralateral superior thyroid artery $\rightarrow$ affected superior thyroid artery $\rightarrow$ external carotid artery $\rightarrow$ neck Internal artery (2 cases); (6) Parathyroid neck $\rightarrow$ superficial cervical 
Table 1 Demographics and blood stealing pathways of 10 patients

\begin{tabular}{|c|c|c|c|c|c|}
\hline NO. & Sex & $\begin{array}{l}\text { Atherosclerotic } \\
\text { risk factors }\end{array}$ & Vascular ultrasound: Blood flow & $\begin{array}{l}\text { CTA: Diagnosis and } \\
\text { stealing pathway }\end{array}$ & $\begin{array}{l}\text { DSA:Diagnosis and } \\
\text { stealing pathway }\end{array}$ \\
\hline 1 & Female & $\mathrm{HT}, \mathrm{DL}$ & $\begin{array}{l}\text { The left CCA had no blood flow, the left ECA had retrograde blood } \\
\text { flow, and the left ICA blood flow decreased but was antegrade. }\end{array}$ & Left CCA occlusion,(12)(3) (4) & Left CCA occlusion,(1)(2)(3)(4) \\
\hline 2 & Male & $\begin{array}{l}\text { Smoke, HT, } \\
\text { DL, DM }\end{array}$ & $\begin{array}{l}\text { The right CCA had no blood flow, the right ECA had retrograde } \\
\text { blood flow, the right ICA blood flow was reduced but antegrade. }\end{array}$ & Right CCA occlusion, (1)(3)(4) & Right CCA occlusion, (1)(3)(4) \\
\hline 3 & Female & HT & $\begin{array}{l}\text { The left CCA had no blood flow, the left ECA had retrograde blood } \\
\text { flow, and the left ICA blood flow decreased but was antegrade. }\end{array}$ & Left CCA occlusion, (1)(2)(3) & Left CCA occlusion, (1)(2)(3) \\
\hline 4 & Male & $\begin{array}{l}\text { Smoke, DL, } \\
\text { DM }\end{array}$ & $\begin{array}{l}\text { The left CCA had no blood flow, the left ECA had retrograde blood } \\
\text { flow, and the left ICA blood flow decreased but was antegrade. }\end{array}$ & $\begin{array}{l}\text { Left CCA occlusion, } \\
\text { (1)(2)(3)(5) }\end{array}$ & $\begin{array}{l}\text { Left CCA occlusion, } \\
\text { (1)(2)(3)(6) }\end{array}$ \\
\hline 5 & Male & $\mathrm{HT}, \mathrm{DL}, \mathrm{DM}$ & $\begin{array}{l}\text { The left CCA had no blood flow, the left ECA had retrograde blood } \\
\text { flow, and the left ICA blood flow decreased but was antegrade. }\end{array}$ & Left CCA occlusion, (1)(3) & Left CCA occlusion, (1)3 \\
\hline 6 & Male & Smoke, HT, DL & $\begin{array}{l}\text { The left CCA had no blood flow, the left ECA had retrograde blood } \\
\text { flow, and the left ICA blood flow decreased but was antegrade. }\end{array}$ & Left CCA occlusion, (2)(3) & Left CCA occlusion, (2)(3) \\
\hline 7 & Male & Smoke, Drink & $\begin{array}{l}\text { The right CCA had no blood flow, the right ECA had retrograde } \\
\text { blood flow, the right ICA blood flow was reduced but antegrade. }\end{array}$ & Right CCA occlusion, (2) & Right CCA occlusion, (2) \\
\hline 8 & Female & HT & $\begin{array}{l}\text { The left CCA had no blood flow, the left ECA had retrograde blood } \\
\text { flow, and the left ICA blood flow decreased but was antegrade. }\end{array}$ & Left CCA occlusion, (2) & Left CCA occlusion, (2) \\
\hline 9 & Male & Smoke, HT, DL & $\begin{array}{l}\text { The right CCA had no blood flow, the right ECA had retrograde } \\
\text { blood flow, the right ICA blood flow was reduced but antegrade. }\end{array}$ & Right CCA occlusion, (1) & Right CCA occlusion, (1) \\
\hline 10 & Male & HT, DL, Drink & $\begin{array}{l}\text { The right CCA had no blood flow, the right ECA had retrograde } \\
\text { blood flow, the right ICA blood flow was reduced but antegrade. }\end{array}$ & Right CCA occlusion, (2) & Right CCA occlusion, (2) \\
\hline
\end{tabular}

Abbreviations: HT Hypertension, DL Dyslipidemia, DM Diabetes mellitus

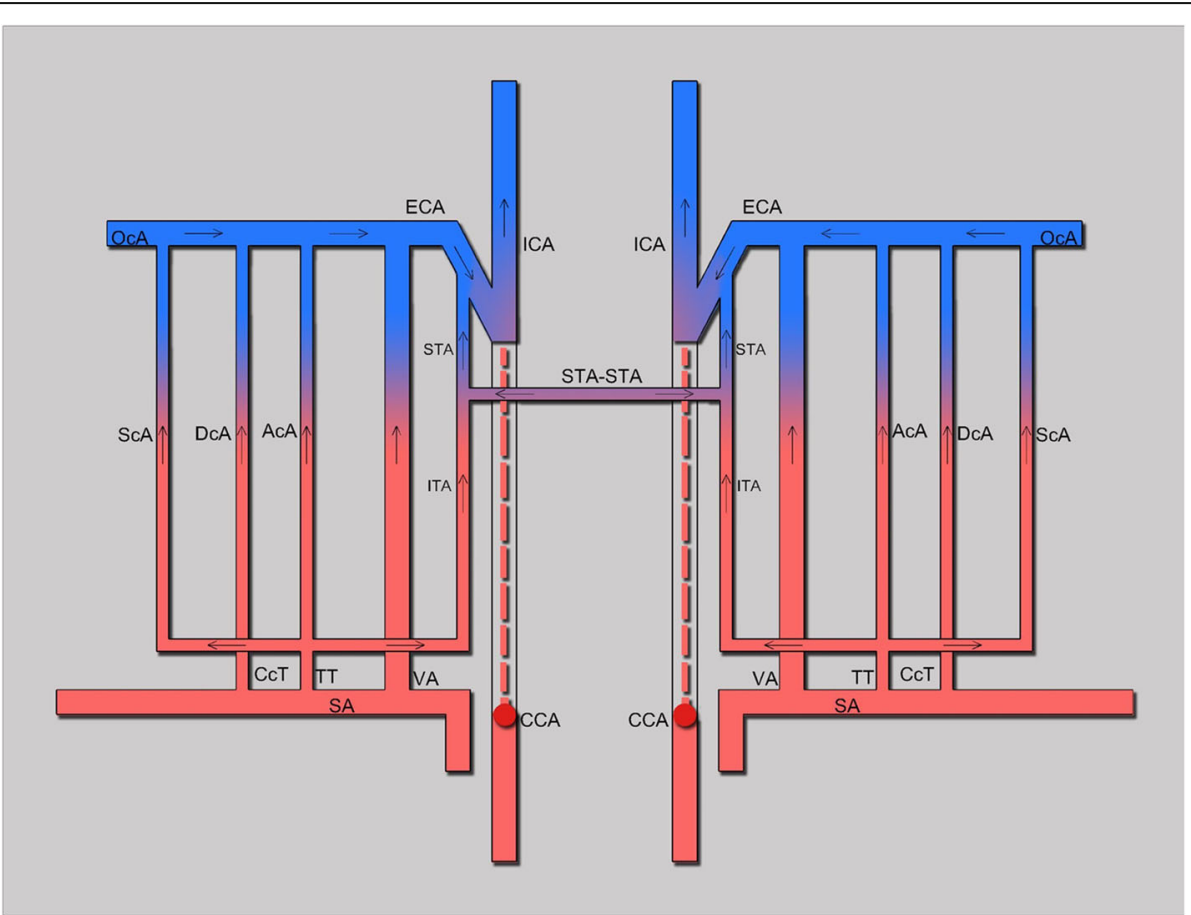

Fig. 1 Schematic diagram of internal carotid artery blood stealing pathways. Abbreviations: CCA: Common carotid artery; ECA: External carotid artery; ICA: Internal carotid artery; SA: Subclavian artery; OcA: Occipital artery; VA: Vertebral artery; TT: Thyrocervical trunk; ACA: Ascending cervical artery; DCA: Deep cervical artery; SCA: Superficial cervical artery; CCT: Costocervical trunk; ITA: Inferior thyroid artery; STA: Superior thyroid artery 


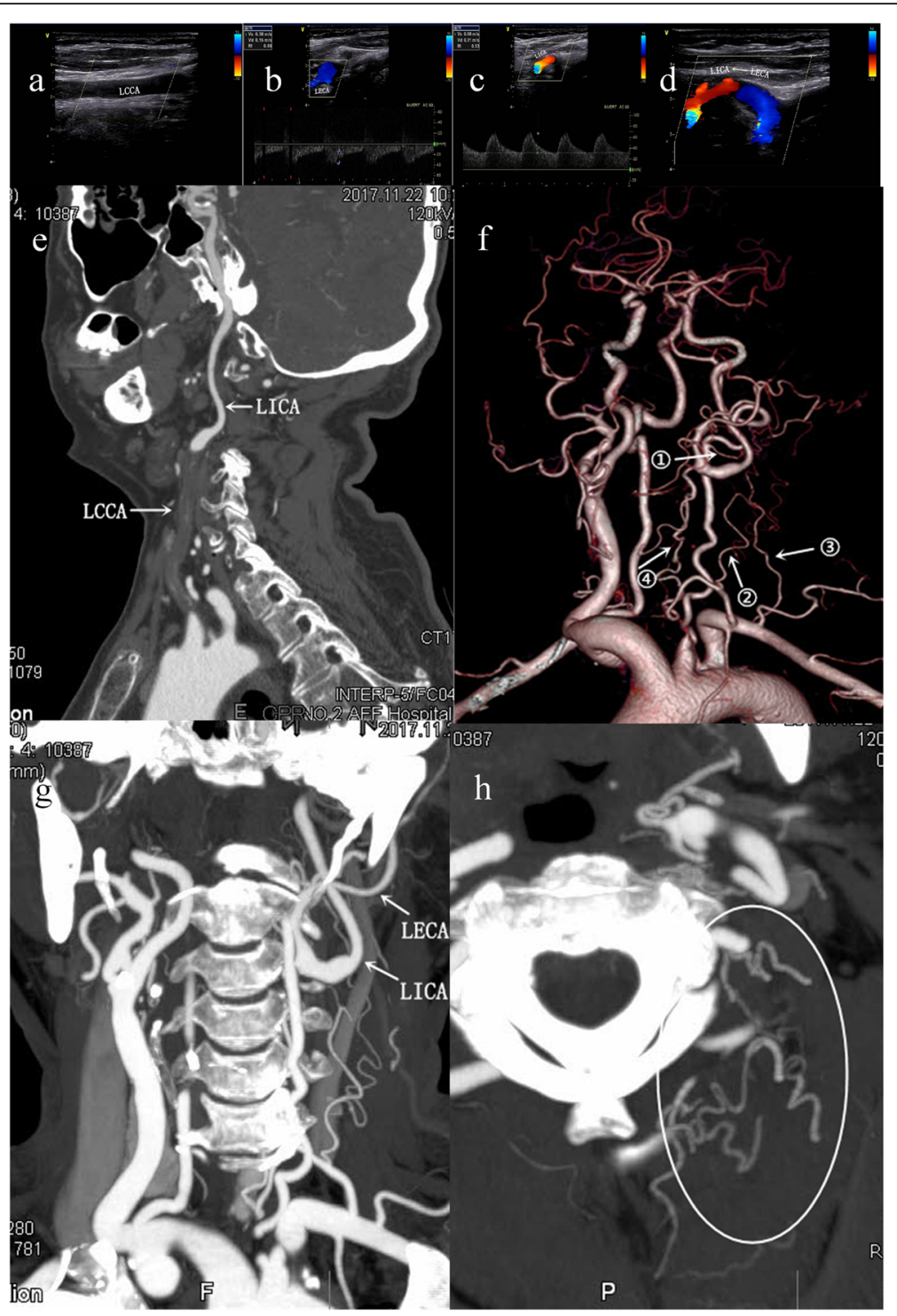

Fig. 2 Patient 1, female. (Fig. 2a-d) Vascular ultrasound shows: a The left CCA had no blood flow, b the left ECA had retrograde blood flow, c the left ICA blood flow, the blood flow spectrum changes with low flow rate and low fluctuation, $\mathbf{d}$ the left ECA supplies blood to the left ICA. (Fig. 2e-h) CTA shows: e Curved Planar Reformation (CPR) shows: The left CCA occlusion, left ICA patency, $\mathbf{f}$ Volume Rendering (VR) shows: internal carotid artery stealing pathway, $\mathbf{g}$ Multi-planner reformation (MPR) shows: the left ICA steals blood access from left ECA, $\mathbf{h}$ Maximal intensity projection (MIP) shows: Deep cervical artery, ascending cervical artery- occipital artery forms the anastomotic vessels

artery $\rightarrow$ occipital artery $\rightarrow$ external carotid artery $\rightarrow$ internal carotid artery (1 case).

\section{Discussion}

CCAOD was a rare clinical disease with clinical manifestations ranging from asymptomatic to severe symptoms of ischemic stroke [8]. The current incidence of CCAOD cannot be accurately assessed [9]. Seker.et al. reported that the prevalence of CCAOD was $0.34 \%$ [10], and other researchers reported that the incidence of CCAOD in patients with ischemic stroke ranges from 0.2 to $4 \%$ [11-13]. These data were consistent with the results of this article. The incidence of CCAOD in this article is $0.39 \%$. In this paper, we found that $55.56 \%$ of CCAOD patients formed a carotid artery stealing pathway with DSA and CTA. The establishment of collateral circulation could guide the treatment and prognosis of stroke $[14,15]$.

In our study, men were more common $(\mathrm{M} / \mathrm{F}=2.33)$. Men were also more common in stroke patients [16]. The results of this article found that left common carotid artery occlusion was more likely to occur $(60 \%)$, and we did not find patients with bilateral common carotid artery occlusion in our study. The risk factors in this article were similar to those in previous literature. 


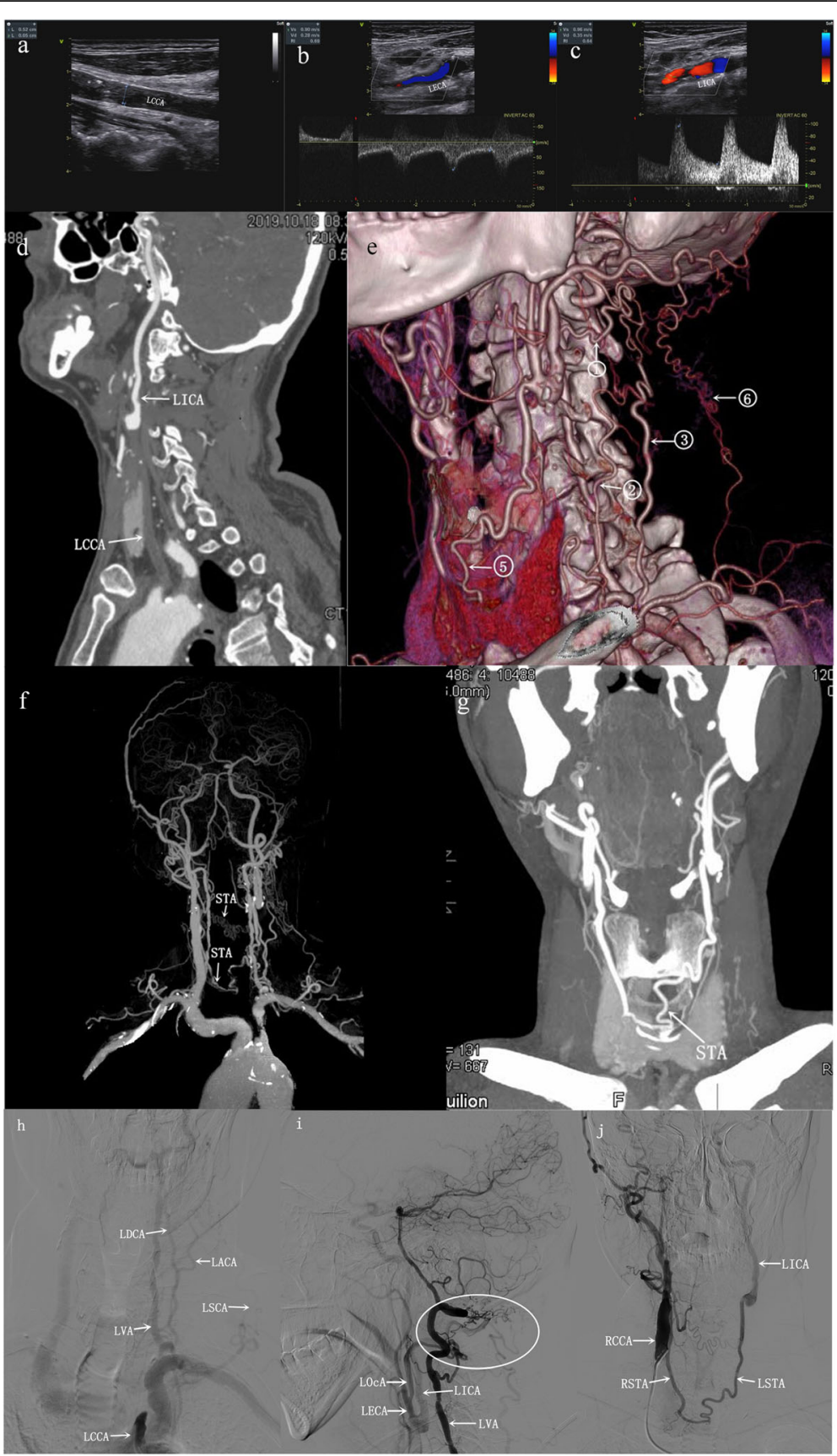

Fig. 3 Patient 4, male. (Fig. 3a-c) Vascular ultrasound shows: a The left CCA had no blood flow, b the left ECA had retrograde blood flow, c left ICA blood flow shows low fluctuations in blood flow spectrum changes, (Fig. 3d-g) CTA shows: $\mathbf{d}$ CPR shows: The left CCA occlusion, left ICA patency, e VR shows: Internal carotid artery stealing pathway, $\mathbf{f} V R$ and $\mathbf{g}$ MIP shows: contralateral superior thyroid artery - affected superior thyroid artery forms an anastomotic blood vessel. (Fig. 3h-j) DSA showed: $\mathbf{h}$ Left superficial cervical artery positive image: the left CCA occlusion, the left ascending cervical artery (ACA), the deep cervical artery (DCA) and the superficial cervical artery (SCA) were thickened and lengthened. $\mathbf{i}$ Left vertebral artery lateral images display stealing pathway: left vertebral artery (VA), left ascending carotid artery (ACA), left deep carotid artery, left superficial carotid artery - left occipital artery (OcA) - left external carotid artery - left internal carotid artery. The circular area indicates that vertebral artery, deep carotid artery, ascending carotid artery and superficial carotid artery form anastomotic vessels. $\mathbf{j}$ Right common carotid artery anteroposterior images display stealing pathway: right common carotid artery - right superior thyroid artery (STA) - left superior thyroid artery - left external carotid artery - left internal carotid artery 
Liebeskind report pointed out that hypertension, hyperlipidemia, diabetes, hypercoagulability and advanced age had a higher prevalence [17]. Our results showed that the prevalence of hypertension was $80 \%$ and the prevalence of hyperlipidemia was $70 \%$. Some literature also reported rare risk factors, such as common carotid dissection, congenital dysplasia, etc.

When an artery in the body waed narrowed or occluded, the pressure at the distal end decreases. A siphonic effect could occur at this time, by "stealing" blood from adjacent vessels through the side branches of the arterial vessel. When the common carotid artery was narrowed or occluded, blood from the external carotid artery was stolen to the internal carotid artery, and we call it internal carotid artery stealing blood. Taking DSA as the gold standard, CTA had a high consistency with DSA in the diagnosis of CCA occlusion, and the coincidence rate was $100 \%$. Analysis of CTA evaluation of internal carotid arty steaming pathways, 6 internal carotid arty steaming pathways were clearly displayed, the coincidence rate of CTA and DSA reached 100\%, the results showed that CTA had a good evaluation effect on collateral circulation path. This is consistent with previous reports. Leng.et al. compared DSA and CTA images of CCAOD, and found that the total coincidence rate of CTA and DSA in judging the degree of vascular stenosis and displaying collateral circulation pathway was 100 and 98\% [12]. DSA has higher spatial resolution, and can directly display collateral circulation and blood flow direction. However, DSA is an invasive examination method. In the process of head and neck angiography, it may lead to serious complications such as arterial dissection, vascular rupture, embolus shedding, etc. DSA is difficult to use in clinical work because of its high technical difficulty, high examination cost and large amount of contrast medium. In contrast, CTA is simple, convenient and inexpensive, and can avoid the occurrence of serious complications. Many literatures have proved that CTA and DSA have high consistency in the diagnosis of head and neck vascular stenosis and the display of collateral circulation pathways [16-18]. DSA can only perform angiography on a single vessel. On the basis of being familiar with the above six internal cardiovascular artery stabilizing pathways, it can avoid the omission of collateral circulation pathway during angiography.

Through DSA and CTA scan of the head and neck, we found 6 collateral circulation pathways for internal carotid stealing blood: (1) Vertebral artery $\rightarrow$ occipital artery $\rightarrow$ external carotid artery $\rightarrow$ internal carotid artery; (2) Thyrocervical trunk $\rightarrow$ ascending cervical artery $\rightarrow$ occipital artery $\rightarrow$ external carotid artery $\rightarrow$ internal carotid artery; (3) Costocervical trunk $\rightarrow$ deep cervical artery $\rightarrow$ occipital artery $\rightarrow$ external carotid artery $\rightarrow$ internal carotid artery;
(4) Affected side thyroid neck trunk $\rightarrow$ inferior thyroid artery $\rightarrow$ superior thyroid artery $\rightarrow$ external carotid artery $\rightarrow$ internal carotid artery; (5) Contralateral external carotid artery $\rightarrow$ contralateral superior thyroid artery $\rightarrow$ affected superior thyroid artery $\rightarrow$ external carotid artery $\rightarrow$ neck Internal artery; (6) Parathyroid neck $\rightarrow$ superficial cervical artery $\rightarrow$ occipital artery $\rightarrow$ external carotid artery $\rightarrow$ internal carotid artery. Verbeeck.et al. reported the pathway (2) through case reports [19], and Lai.et al. reported the pathway (3) through case reports [1]. CCAOD could provide blood perfusion to the diseased brain through 6 pathways. The results of this article showed that $60 \%$ of patients found pathway (1), 70\% of patients found pathway (2), and $60 \%$ of patients found pathway (3). The occipital artery pathway was found in all patients, it can be deduced that the occipital artery pathway was the most important pathway for CCAOD to relieve cerebral blood perfusion. The results of this paper found that most internal carotid artery blood flow originated from the ipsilateral blood vessels. Only one case originated from the contralateral external carotid artery. So far, no related literature has reported six pathways of internal carotid blood stealing. DSA was the gold standard for diagnosing vascular disease. However, in clinical work, non-invasive vascular ultrasound and CTA tests were more practical [1]. The patients in this article were diagnosed with CCAOD and internal carotid artery steal by DSA. The display of collateral circulation pathways by neck vascular ultrasound was not as clear, intuitive, and accurate as CTA scans of the head and neck [20].

\section{Conclusions}

The incidence of CCAOD is very low, and the blood stealing pathway is complicated. Common risk factors were hypertension and hyperlipidemia. We could use head and neck CTA to discover the internal carotid blood stealing pathway.

\begin{abstract}
Abbreviations
CCA: Common carotid artery; CCAOD: Common carotid artery occlusion disease; CTA: Computed tomography angiography; DSA: Digital subtraction angiography; ECA: External carotid artery; ICA: Internal carotid artery; SA: Subclavian artery; OcA: Occipital artery; VA: Vertebral artery;

TT: Thyrocervical trunk; ACA: Ascending cervical artery; DCA: Deep cervical artery; SCA: Superficial cervical artery; CCT: Costocervical trunk; ITA: Inferior thyroid artery; STA: Superior thyroid artery; VR: Volume rendering; MPR: Multiplanner reformation; MIP: Maximum intensity projection; CPR: Curved planar reconstruction
\end{abstract}

\section{Acknowledgements}

None.

Authors' contributions

Conception and design: YGW and DXW; Acquisition the materials and data: ZL and XYZ; Analysis and interpretation of data DXW, HYC, ZHW; Drafted the manuscript DXW; JH and TYZ critically revised the manuscript for important intellectual content. All authors have read and approved the manuscript. 


\section{Funding}

This work was supported by Clinical Research Fund of Qiqihar Medical Academy (QMSI2019L-14). The funders had no role in study design, data collection and analysis, decision to publish, or preparation of the manuscript.

\section{Availability of data and materials}

The datasets used and analyzed during the present study are available from the corresponding author on reasonable request.

\section{Ethics approval and consent to participate}

The study protocol was approved by the Regional Ethics Committee for Clinical Research of the Second Affiliated Hospital of Qiqihar Medical College (Qiqihar, 161006, China). All patients have signed informed consent.

\section{Consent for publication}

Not applicable.

\section{Competing interests}

The authors have declared that no competing interests exist.

\section{Author details}

${ }^{1}$ Departments of CT, The Second Affiliated Hospital of Qiqihar Medical College, 37 West Zhonghua Road, Qiqihar, Heilongjiang 161006, PR China. 2Department of Electrophysiology, The Second Affiliated Hospital of Qiqihar Medical College, Qiqihar 161006, Heilongjiang, China. ${ }^{3}$ Department of Anatomy, Qiqihar Medical College, Qiqihar 161006, Heilongjiang, China.

Received: 11 June 2020 Accepted: 30 August 2020

Published online: 03 September 2020

\section{References}

1. Lai S-L, Chen Y-C, Weng H-H, Chen S-T, Hsu S-P, Lee T-H. Bilateral common carotid artery occlusion-a case report and literature review. J Neurol Sci. 2005;238(1-2):101-4..

2. Bang OY, Saver JL, Kim SJ, et al. Collateral flow predicts response to endovascular therapy for acute ischemic stroke. Stroke. 2011;42:693-9.

3. Goyal M, Demchuk AM, Menon BK, et al. Randomized assessment of rapid endovascular treatment of ischemic stroke. N Engl J Med. 2015;372:1019-30.

4. Fanou EM, Knight J, Aviv Rl, et al. Effect of collaterals on clinical presentation, baseline imaging, complications, and outcome in acute stroke. AJNR Am J Neuroradiol. 2015;36:2285-91.

5. Beyer SE, Thierfelder KM, von Baumgarten $L$, et al. Strategies of collateral blood flow assessment in ischemic stroke: prediction of the follow-up infarct volume in conventional and dynamic CTA. AJNR Am J Neuroradiol. 2015;36:488-94.

6. Vagal A, Menon BK, Foster LD, et al. Association between CT angiogram collaterals and CT perfusion in the interventional Management of Stroke III trial. Stroke. 2016:47:535-8.

7. Albers GW, Marks MP, Kemp S, Marks SK, et al. Thrombectomy for stroke at 6 to 16 hours with selection by perfusion imaging. N Engl J Med. 2018;378: 708-18.

8. Wang $Y$, Wang $Y$, Zhao $X$, et al. Clopidogrel with aspirin in acute minor stroke or transient ischemic attack. N Engl J Med. 2013;369:11-9.

9. Kim JM, Jung $\mathrm{KH}$, Sohn $\mathrm{CH}$, et al. Intracranial plaque enhancement from high resolution vessel wall magnetic resonance imaging predicts stroke recurrence. Int J Stroke. 2016;11:171-9.

10. Seker F, Potreck A, Möhlenbruch M, et al. Comparison of four different collateral scores in acute ischemic stroke by CT angiography. J Neurointerv Surg. 2016;8:1116-8.

11. Powers WJ, Derdeyn CP, Biller J, et al. American Heart Association/American Stroke Association focused update of the 2013 guidelines for the early management of patients with acute ischemic stroke regarding endovascular treatment: a guideline for healthcare professionals from the American Heart Association/American Stroke Association. Stroke. 2015:2015:3020-35.

12. Leng $X$, Lan L, Liu L, et al. Good collateral circulation predicts favorable outcomes in intravenous thrombolysis: a systematic review and metaanalysis. Eur J Neurol. 2016;23:1738-49.

13. Saver JL, Goyal M, Bonafe A, et al. Stent-retriever thrombectomy after intravenous t-PA vs. t-PA alone in stroke. N Engl J Med. 2015;372:2285-95.

14. Jovin TG, Saver JL, Ribo $M$, et al. Diffusion-weighted imaging or computerized tomography perfusion assessment with clinical mismatch in the triage of wake up and late presenting strokes undergoing neurointervention with Trevo (DAWN) trial methods. Int J Stroke. 2017;12: 641-52.

15. De Silva KR, Silva R, Amaratunga $D$, et al. Types of the cerebral arterial circle (circle of Willis) in a Sri Lankan population. BMC Neurol. 2011;11:5.

16. Leng $X$, Fang $H$, Leung TW, et al. Impact of collateral status on successful revascularization in endovascular treatment: a systematic review and metaanalysis. Cerebrovasc Dis. 2016:41:27-34.

17. Liebeskind DS. Collateral lessons from recent acute ischemic stroke trials. Neurol Res. 2014;36:397-402.

18. Liggins JT, Mlynash $\mathrm{M}$, Jovin $\mathrm{TG}$, et al. Interhospital variation in reperfusion rates following endovascular treatment for acute ischemic stroke. J Neurointerv Surg. 2015;7:231-3.

19. Verbeeck NY, Vazquez Rodriguez C. Patent internal and external carotid arteries beyond an occluded common carotid artery: report of a case diagnosed by color Doppler. J Belg Radiol. 1999;82(5):219-21.

20. Lee WJ, Jung KH, Ryu YJ, et al. Utility of digital subtraction angiographybased collateral evaluation in medically treated acute symptomatic basilar artery stenosis. Eur J Neurol. 2017;24:1148-55.

\section{Publisher's Note}

Springer Nature remains neutral with regard to jurisdictional claims in published maps and institutional affiliations.

Ready to submit your research? Choose BMC and benefit from:

- fast, convenient online submission

- thorough peer review by experienced researchers in your field

- rapid publication on acceptance

- support for research data, including large and complex data types

- gold Open Access which fosters wider collaboration and increased citations

- maximum visibility for your research: over $100 \mathrm{M}$ website views per year

At BMC, research is always in progress.

Learn more biomedcentral.com/submissions 